\title{
CALIDAD DE VIDA RELACIONADA CON LA SALUD ORAL EN ADULTOS DE LA CIUDAD DE MACAS, ECUADOR, 2021
}

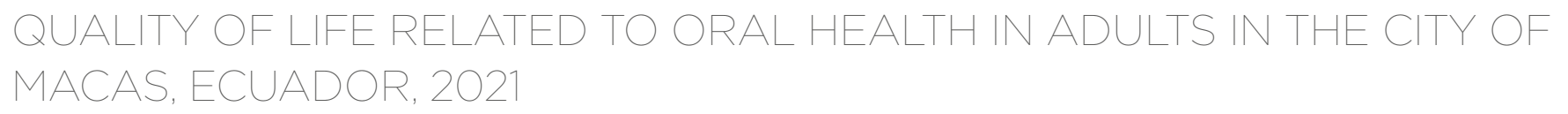

Jefferson Israel Molina-Merino' * (i) jeffersonisra.7@gmail.com

María del Cisne Centeno-Dávila2 (i) mcentenod@ucacue.edu.ec

Artículo recibido: 19/08/2021

Arbitrado por pares

Artículo aceptado: 16/09/2021

Artículo publicado: 12/10/2021

* Autor corresponsal:

Jefferson Israel Molina Merino

jeffersonisra.7@gmail.com

\section{(a) (i)}

CLos autores, 2021. Publicado por la Universidad Científica del Sur (Lima, Perú)

\section{RESUMEN}

Objetivo: Determinar la calidad de vida relacionada con la salud bucal en las personas de 18 a 99 años en la ciudad de Macas, Ecuador, en 2021. Materiales y métodos: El estudio fue descriptivo, de corte transversal, con un enfoque cuantitativo. Se evaluó a 415 personas mayores de 18 años pertenecientes a la ciudad de Macas. Para medir este estudio, se utilizó el instrumento OHIP-14, compuesto por 7 dimensiones, cada una con 2 preguntas. Para la medición de las variables (sexo, edad, nivel socioeconómico, ocupación), se aplicó la prueba estadística U de Mann-Withney y p $<0,05$. Resultados: Se demostró un predominio en el sexo femenino, con un 63\%. La dimensión con alto impacto de manera general de todos los encuestados es malestar psicológico. En las puntuaciones dimensionales del OHIP-14 entre hombres y mujeres se encontraron diferencias estadísticamente significativas en limitación funcional y minusvalía, de $\mathrm{p}=0,012$ y $\mathrm{p}=0,036$ Conclusiones: Existe una relación entre la calidad de vida y la salud oral de los habitantes de la ciudad de Macas. El OHIP-14 muestra la dimensión de malestar psicológico como el principal factor de los problemas orales.

Palabras clave: calidad de vida, salud oral, adulto

\section{ABSTRACT}

Objective: To determine the quality of life related to oral health in 18-99-year-old people in the city of Macas-Ecuador 2021. Materials and methods: This was a descriptive, cross-sectional study following a quantitative approach. A total of 415 people over 18 years of age from Macas-Ecuador were evaluated. The instrument of measure was a OHIP-14 instrument composed of 7 dimensions, each of which had 2 questions. The study variables (sex, age, socioeconomic level, occupation) were analyzed using the Mann-Whitney $U$ statistical test, with $\mathrm{p}<0.05$ being considered as significant. Results: There was a predominance of $63 \%$ of women. The dimension of "psychological distress" had the highest overall impact of all the items answered. Comparison of the OHIP-14 dimensional scores between men and women showed statistically significant differences in functional limitation and handicap with $p=0.012$ and $p=0.036$, respectively. Conclusions: There is a relationship between quality of life and oral health among the inhabitants of the city of Macas-Ecuador. The OHIP-14 instrument showed the dimension of "psychological distress" to be the main factor of oral problems.

Keywords: Quality of life, oral health, adult

Citar como: Molina-Merino J, CentenoDávila MC. Calidad de vida relacionada con la salud oral en adultos de la ciudad de Macas, Ecuador, 2021. Rev Cient Odontol (Lima). 2021; 9(3): e068.

DOI: 10.21142/2523-2754-0903-2021-068

1 Carrera de Odontología, Universidad Católica de Cuenca. Cuenca, Ecuador.

2 División de Periodoncia e Implantología Quirúrgica, Carrera de Odontología, Universidad Católica de Cuenca. Cuenca, Ecuador. 


\section{INTRODUCCIÓN}

Se describe la calidad de vida como el bienestar del individuo, teniendo en cuenta el estilo de vida, el lugar donde convive, la eficiencia de la educación, el medio laboral y el estado económico. La OMS, en 1966, definió la calidad de vida como "la percepción del individuo sobre su posición en la vida dentro del contexto cultural $\mathrm{y}$ sistema de valores en que vive, y con respecto a sus objetivos, expectativas, estándares e intereses” $\left.{ }^{1,2}\right)$.

Los diversos cambios que le ocurren a un individuo durante su vida influyen directamente en su calidad de vida, por ello se han realizado distintas campañas, mediante encuentros o publicidad, que promueven la prevención y el cuidado de la salud, con la finalidad de obtener calidad de vida a través del cuidado personal $\left({ }^{3}\right)$.

La calidad de vida relacionada con la salud oral (CVRSO) está determinada por la interacción entre las condiciones bucales y los problemas cotidianos. La OMS indica que la salud bucodental interactúa con la salud física, psicológica y social, así como con los tejidos blandos y duros presentes en la cavidad oral, por lo que existe relación entre el cuidado dental y una buena calidad de vida $(2,4)$.

Por tanto, la salud oral trata de buscar la razón de la falta de preocupación en la higiene bucal de las personas, e intenta generar un cambio positivo para que el paciente acuda a la atención odontológica antes de que se produzca una enfermedad, molestia o dolor. Actualmente, las personas solo acuden al dentista por dolor o, en ciertos casos, por estética, por lo que muestran un bajo cuidado de la salud oral $\left({ }^{5,6}\right)$.

Se ha observado que la caries dental, junto con la enfermedad periodontal, son los factores más recurrentes en la cavidad bucal, por lo que se encuentra en la tercera posición de las enfermedades a nivel mundial y ocasiona intranquilidad en la vida diaria de las personas. Se han descrito otras patologías bucales que influyen en la calidad de vida, como la presencia de fluorosis o manchas, las alteraciones de los tejidos dentarios, las maloclusiones, los traumatismos, las anomalías craneofaciales y el uso de prótesis dentales $\left({ }^{9,11}\right)$.
Para la medición de la CVRS, Slade et al. $\left(^{7}\right)$ validaron el instrumento OHIP-49 para la medición de la calidad de vida en relación con la salud bucal. Este instrumento, inicialmente, estaba conformado por un cuestionario de 49 preguntas, pero en 1997 se modificó a OHIP-14, que es una variante de 14 preguntas. Este cuestionario mide el impacto de los problemas bucales de las personas y cómo influyen en su entorno, y logra determinar los problemas funcionales, sociales y psicológicos que padece el individuo, lo cual demuestra su confiablidad. Estos 14 ítems se basan en 7 dimensiones: limitación funcional, malestar físico, malestar psicológico, discapacidad física, discapacidad psicológica, discapacidad social y minusvalía. En Latinoamérica, Montero et al. $\left(^{8}\right)$ adaptaron el instrumento OHIP-14 a una versión en español: el OHIP-14sp.

Se desconocen estudios previos sobre la CVRSO en la ciudad de Macas, Ecuador; por esta razón, el objetivo del presente estudio es determinar la calidad de vida relacionada con la salud bucal en las personas de 18 a 99 años en la ciudad de Macas, en 2021.

\section{MATERIALES Y MÉTODOS}

Se realizó un estudio descriptivo, de corte transversal, con un enfoque cuantitativo. Este estudio se analizó en el periodo 2021, en la ciudad de Macas, Ecuador, que tiene 19,176 habitantes. De esta población, se obtuvieron una muestra de 415 personas, utilizando la formula general de estudios descriptivos, con un intervalo de confianza del 95\%.

Los criterios de inclusión fueron personas residentes de la ciudad de Macas y que tengan edades entre los 18 y 99 años, sin distinción de sexo.

Los criterios de exclusión fueron personas que no respondieron correctamente las preguntas y sobre todo que no hubieran completado la encuesta.

El instrumento que se utilizó fue la versión en español del OHIP-14, que fue validada por Montero $\left({ }^{8}\right)$ y se 
utiliza para la medición de la CVRSO; variante más corta, pero de igual exactitud y precisión que la OHIP49 , ambas validadas por Slade $\left({ }^{7}\right)$.

Los datos obtenidos fueron recogidos en línea, mediante encuestas elaboradas en la plataforma Microsoft Forms y enviadas mediante la aplicación WhatsApp a las personas residentes de la ciudad de Macas, ya que no se pudieron realizar de forma presencial dado el confinamiento por el virus SARS-CoV-2.

Para la medición de las variables (sexo, edad, nivel socioeconómico, ocupación), se utilizó la prueba estadística U de Mann-Withney, mientras que para la revisión, tabulación y análisis de los resultados se utilizaron valores de frecuencia absoluta y porcentual, mediante el programa estadístico IBM SPSS Statitics v.26.

\section{RESULTADOS}

Del total de 415 personas encuestadas, el 63\% corresponde al sexo femenino. Los habitantes de 18 a 44 años predominaron en el grupo de edad, con un $83 \%$. En cuanto al nivel de instrucción en la muestra predominan las personas con educación superior, con un 42,2\%, seguidas por las que tienen secundaria completa, con un 40\% (tabla 1).

Tabla 1. Distribución de la muestra de acuerdo con sexo/ edad y nivel de instrucción

\begin{tabular}{|c|c|c|c|c|c|c|}
\hline \multicolumn{7}{|c|}{ Sexo } \\
\hline \multirow{2}{*}{ Grupo de edad } & \multicolumn{2}{|c|}{ Femenino } & \multicolumn{2}{|c|}{ Masculino } & \multicolumn{2}{|c|}{ Total } \\
\hline & n & $\%$ & n & $\%$ & $\mathbf{n}$ & $\%$ \\
\hline De 18 a 44 años & 217 & $83 \%$ & 128 & $83 \%$ & 345 & $83 \%$ \\
\hline De 45 a 64 años & 38 & $15 \%$ & 21 & $14 \%$ & 59 & $14 \%$ \\
\hline De 65 a más años & 6 & $2 \%$ & 5 & $3 \%$ & 11 & $3 \%$ \\
\hline Total & 261 & $100 \%$ & 154 & $100 \%$ & 415 & $100 \%$ \\
\hline \multicolumn{7}{|c|}{ Nivel de instrucción } \\
\hline & & & \multicolumn{2}{|c|}{$\mathbf{n}$} & \multicolumn{2}{|c|}{$\%$} \\
\hline Primaria & & & 11 & & 2,7 & \\
\hline Secundaria & & & 166 & & 40,0 & \\
\hline Superior Técnico & & & 63 & & 15,2 & \\
\hline Superior Universitario & & & 175 & & 42,2 & \\
\hline Total & & & 415 & & 100,0 & \\
\hline
\end{tabular}

Respecto del ingreso económico, el grupo de menores ingresos al mes fue el de amas de casa (42\%) y el de mayores ingresos, el de los profesionales (32\%) (tabla 2).

Más del 50\% de los encuestados manifestaron un bajo o nulo malestar funcional en su estado de salud oral, mientras que el malestar psicológico fue el más predominante entre los pobladores (tabla 3 ).

Las preguntas que demostraron mayor impacto fueron las relacionadas con malestar psicológico, discapacidad física, discapacidad psicológica, discapacidad social y minusvalía; solo la limitación funcional no mostró un gran impacto (tabla 4).

En los puntajes de las dimensiones del OHIP-14 entre varones y mujeres se encontraron diferencias estadísticamente significativas en limitación funcional, con $\mathrm{p}=0,012$, y minusvalía, con $\mathrm{p}=0,036$, en ambos casos con peor puntaje para el sexo masculino (tabla 5).

Tabla 2. Distribución de la muestra de acuerdo con nive de ingreso (dólares) y ocupación

\begin{tabular}{|c|c|c|c|c|c|}
\hline \multirow[t]{2}{*}{ Ocupación } & \multicolumn{2}{|c|}{$\begin{array}{c}400 \text { dólares o } \\
\text { menos por persona }\end{array}$} & \multicolumn{2}{|c|}{$\begin{array}{c}\text { Mayor a } 400 \\
\text { dólares por persona }\end{array}$} & \multirow{2}{*}{$\begin{array}{c}\text { Total } \\
\mathbf{n}\end{array}$} \\
\hline & $n$ & $\%$ & n & $\%$ & \\
\hline $\begin{array}{l}\text { Agricultor, pescador, } \\
\text { ganadero }\end{array}$ & 11 & $3 \%$ & 4 & $4 \%$ & 13 \\
\hline $\begin{array}{l}\text { Ama de casa, estudiante } \\
\text { o trabajador no } \\
\text { calificado }\end{array}$ & 137 & $42 \%$ & 25 & $28 \%$ & 166 \\
\hline Desempleado & 54 & $17 \%$ & 5 & $6 \%$ & 59 \\
\hline $\begin{array}{l}\text { Empleado de oficina / } \\
\text { Apoyo administrativo }\end{array}$ & 44 & $14 \%$ & 13 & $14 \%$ & 57 \\
\hline $\begin{array}{l}\text { Gerente o miembro del } \\
\text { Poder Ejecutivo }\end{array}$ & 1 & $0 \%$ & 3 & $3 \%$ & 4 \\
\hline Militar o policía & 3 & $1 \%$ & 0 & $0 \%$ & 3 \\
\hline $\begin{array}{l}\text { Operador de } \\
\text { plantas máquinas y } \\
\text { ensambladores }\end{array}$ & 4 & $1 \%$ & I & $1 \%$ & 5 \\
\hline $\begin{array}{l}\text { Operario o artesano y } \\
\text { oficios conexos }\end{array}$ & 14 & $4 \%$ & 0 & $0 \%$ & 14 \\
\hline $\begin{array}{l}\text { Profesional, científico } \\
\text { o intelectual de libre } \\
\text { ejercicio }\end{array}$ & 17 & $5 \%$ & 29 & $32 \%$ & 46 \\
\hline $\begin{array}{l}\text { Trabajador de servicios } \\
\text { y ventas (comerciante) }\end{array}$ & 40 & $12 \%$ & 11 & $12 \%$ & 48 \\
\hline Total & 325 & $100 \%$ & 90 & $100 \%$ & 415 \\
\hline
\end{tabular}


Tabla 3. Frecuencia del índice OHIP-14 con respecto a los encuestados

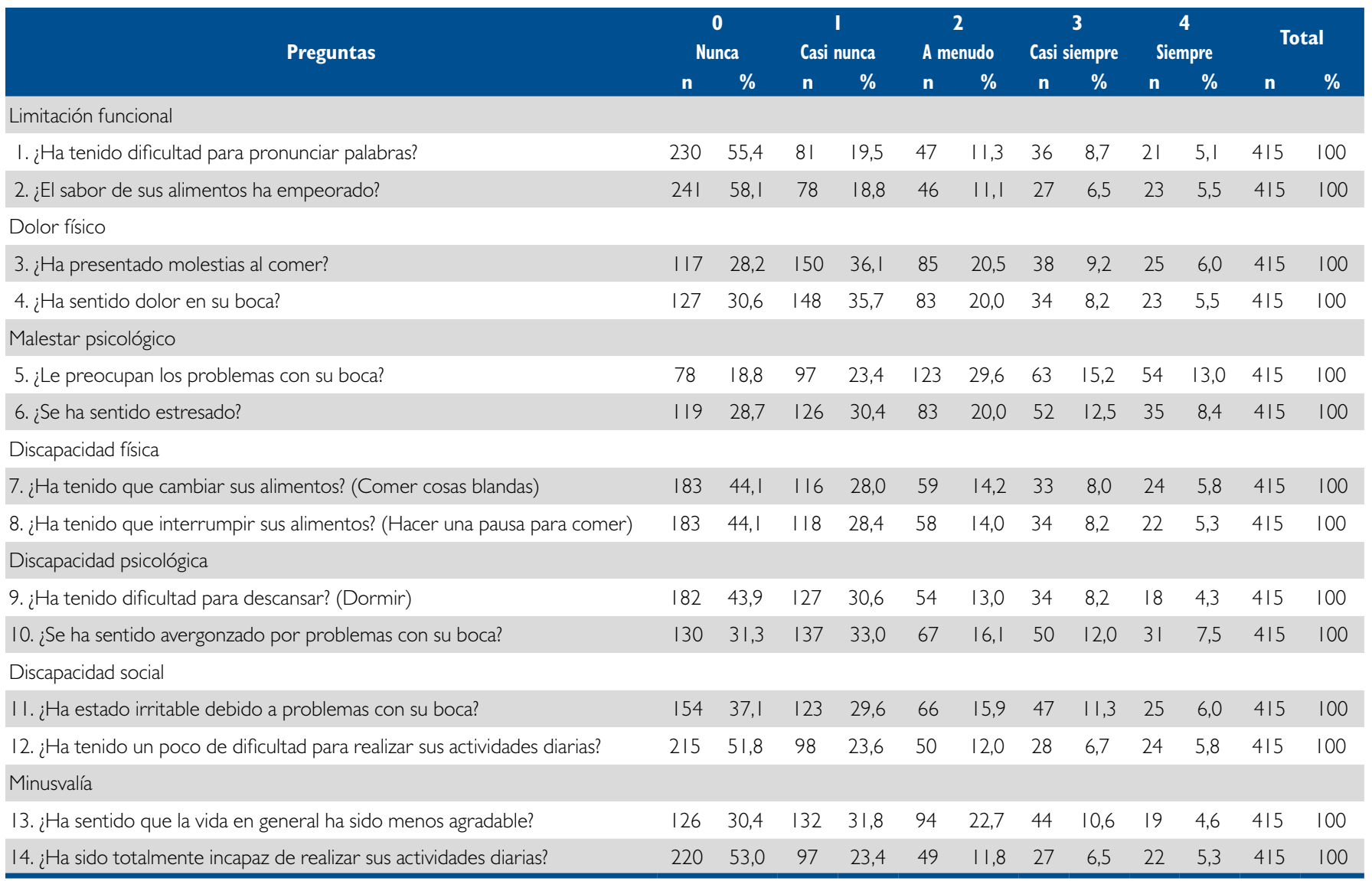

\section{DISCUSIÓN}

Al culminar la investigación sobre la calidad de vida relacionada con la salud bucal en la ciudad de Macas, Ecuador, se halló un predominio en el sexo femenino, con un 63\%, similar a los resultados de Jenei et al. $\left({ }^{12}\right)$, en su estudio realizado para medir la CVRSO en personas que asistieron a la Facultad de Odontología de la Universidad de Debrecen (Hungría), con un 65,5\%. Por el contrario, en el estudio de Mohamed et al. $\left({ }^{14}\right)$, se menciona un predominio en el sexo masculino, con un 58,7\%; esto se explicar porque el estudio se realizó al sur de Asia, en un hospital donde las mujeres parecen tener menos accesibilidad en la atención médica.

Con respecto a los intervalos de edad, se obtuvo que los encuestados de 18 a 44 años, que corresponden a adultos jóvenes, presentaron mayor incidencia, con un $83 \%$, lo que concuerda con el estudio de Husain y Tatengkeng $\left({ }^{4}\right)$.
El nivel de instrucción más prevalente en la ciudad de Macas fue el "Superior Universitario", con un 42\%. En el estudio de Utsman et al. $\left({ }^{13}\right)$, realizado en la ciudad de San José (Costa Rica), se obtuvo el mismo valor. En la investigación de Papaioannou et al. $\left({ }^{19}\right)$, realizado en Grecia, se indica que la "Secundaria" fue la más prevalente, con un $41,4 \%$, mientras que la secundaria ocupó el segundo puesto, con un 40\%. Esto indicaría que las personas latinas se preocupan más por su nivel de educación.

Los ingresos mensuales mencionados por Husain y Tatengkeng ( ${ }^{4}$ ) mostraron que el 79,2\% gana valores menores a 500 dólares al mes, resultado similar al del presente estudio, el cual halló que el $78.5 \%$ están por debajo de los 400 dólares de ingreso mensual. 
Tabla 4. Agrupación de las preguntas de OHIP-14 con respecto a su impacto y no impacto

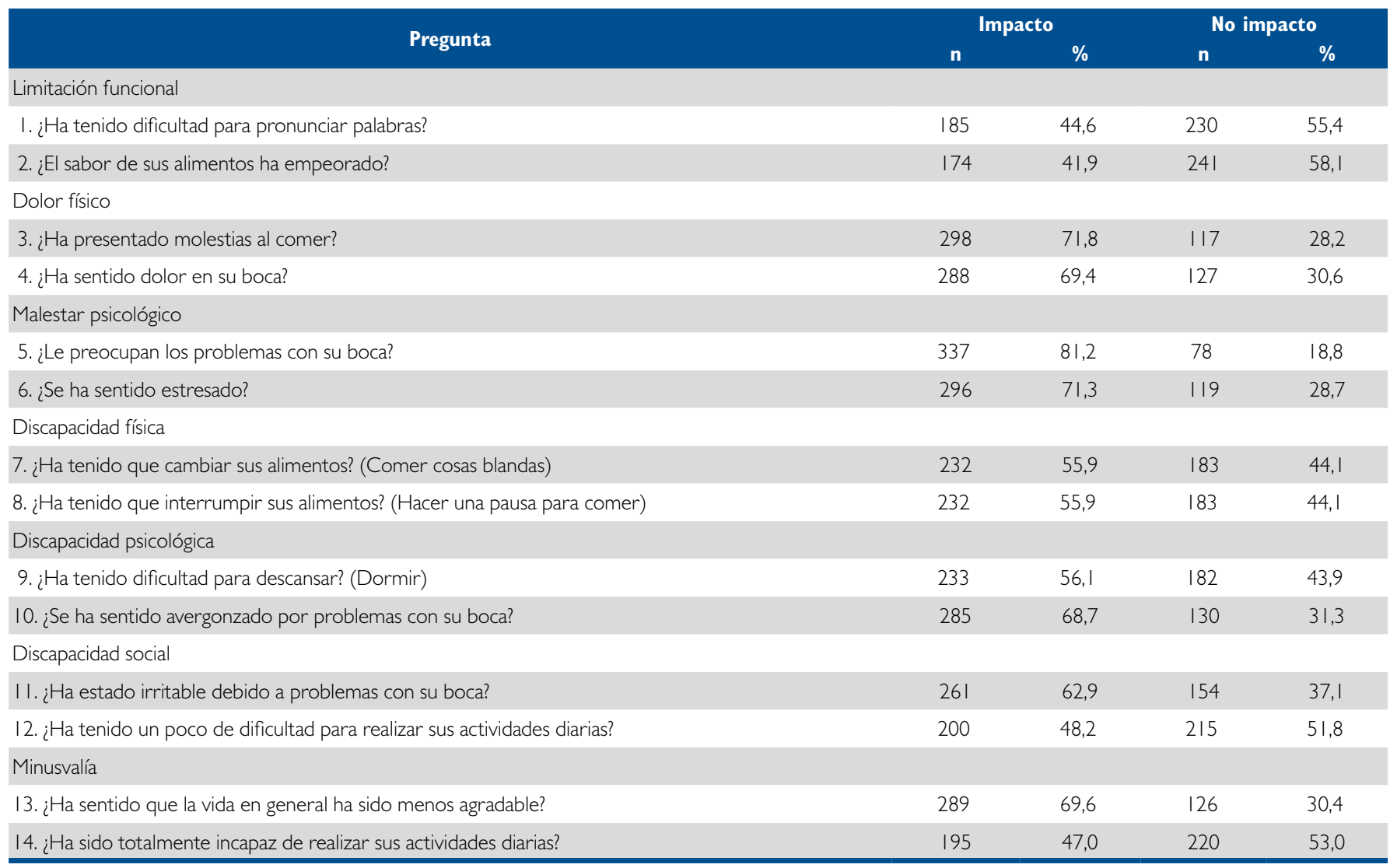

Tabla 5. Puntaje de las dimensiones del OHIP-14 con respecto al sexo

\begin{tabular}{lccccccc} 
& \multicolumn{2}{c}{ Femenino } & \multicolumn{2}{c}{ Masculino } & \multicolumn{2}{c}{ Total } & P \\
& Media & D. E. & Media & D. E. & Media & D. E. \\
\hline Limitación funcional & 1,5 & 2,1 & 2,1 & 2,4 & 1,7 & 2,2 & 0,012 \\
Dolor físico & 2,4 & 2,1 & 2,7 & 2,2 & 2,5 & 2,2 & 0,273 \\
Malestar psicológico & 3,1 & 2,3 & 3,4 & 2,2 & 3,2 & 2,3 & 0,138 \\
Incapacidad física & 2,1 & 2,1 & 2,5 & 2,4 & 2,2 & 2,2 & 0,151 \\
Incapacidad psicológica & 1,8 & 2,1 & 2,3 & 2,4 & 2,0 & 2,2 & 0,080 \\
Incapacidad social & 2,0 & 2,1 & 2,4 & 2,4 & 2,2 & 2,2 & 0,168 \\
Minusvalía & 2,0 & 2,0 & 2,5 & 2,4 & 2,2 & 2,2 & 0,036 \\
\hline
\end{tabular}

Prueba de $U$ de Mann-Whitney

Mohamed et al., Rodakowska et al.y Koistinen et al. (14,20, ${ }^{21}$ ) refieren mayor prevalencia en el malestar psicológico con respecto al cuestionario OHIP-14, lo que muestra similitud con este estudio. Además, Henriques et al. $\left({ }^{16}\right)$ y Gaber et al. $\left({ }^{17}\right)$ indican mayor incidencia en el dolor físico, lo que se puede corroborar ya que las personas fueron atendidas y encuestadas en una clínica odontológica.
Silva et al. $\left({ }^{15}\right)$ describieron el malestar psicológico, el dolor físico y la discapacidad psicológica como las dimensiones de mayor impacto en las personas, tal como en este estudio. Mohamed et al. $\left({ }^{14}\right)$ mencionan como dimensión de menor impacto a la discapacidad social, mientras en el presente estudio el de menor impacto fue la limitación funcional. Esto porque en la presente investigación las personas encuestadas no han tenido 
problemas al pronunciar palabras ni tenido mal sabor al ingerir sus alimentos.

En los puntajes de las dimensiones del OHIP-14 entre hombres y mujeres del presente estudio, se encontraron diferencias estadísticamente significativas en limitación funcional y minusvalía, compatibles con las investigaciones de Hanisch et al. $\left({ }^{18}\right)$ y Acharya $\left({ }^{22}\right)$.

De acuerdo con varios estudios, los descritos son factores sobre los que se debe adquirir mayor conciencia por parte de los profesionales de la salud oral, para ayudar a la promoción y prevención, y a que las personas tengan una mejor calidad de vida. Los resultados de este estudio ayudarán a futuras investigaciones.

\section{CONCLUSIÓN}

Se determinó la relación entre la calidad de vida y la salud oral de los habitantes residentes de la ciudad de Macas, Ecuador, en 2021, y se halló que el malestar psicológico constituye la mayor problemática de manera general entre los encuestados. Al comparar por sexo, el masculino tuvo diferencias estadísticamente significativas en limitación funcional y minusvalía.

Contribución de autoría: Jefferson Molina Merino ha participado en la concepción, la recolección de datos, el análisis e interpretación de datos, la redacción y la aprobación de la versión final del artículo. María del Cisne Centeno ha contribuido como asesora en el diseño del trabajo, la interpretación de los datos, la redacción y la aprobación final del artículo.

Potenciales conflictos de intereses: Los presentes autores no manifiestan ningún conflicto de intereses.

Fuente de financiamiento: Autofinanciado. 


\section{REFERENCIAS BIBLIOGRÁFICAS}

1. Díaz S, Tirado L, Tamayo G. Impacto de la sonrisa sobre calidad de vida relacionada con salud bucal en adultos. Rev. Clin. Periodoncia Implantol. Rehabil. Oral. 2018; 11(2): 78-83. doi: http://dx.doi.org/10.4067/S0719-01072018000200078

2. Velázquez L, Ortiz L, Cervantes A, Cárdenas A, García C, Sánchez S. Calidad de vida relacionada con la salud oral en adultos mayores. Instrumentos de evaluación. Rev Med Inst Mex Seguro Soc. 2014; 52(4): 448-56.

3. Vélez V, Villavicencio E, Cevallos S, Del Castillo C. Impacto de la calidad de vida relacionada a la salud bucal; Machángara, Ecuador. Estomatológica Heredia. 2019: 29(3): 203-212. doi: http://dx.doi.org/10.20453/reh.v29i3.3604

4. Husain F, Tatengkeng F. Oral Health-Related Quality of Life Appraised by OHIP-14 Between Urban and Rural Areas in Kutai Kartanegara Regency, Indonesia: Pilot Pathfinder Survey. Open Dent J. 2017; 11: 557-64. doi: https://doi. org/10.2174/1874210601711010557

5. Diaz C. V, Casas I, Roldán J. Calidad de vida relacionada con salud oral: Impacto de diversas situaciones clínicas odontológicas y factores socio-demográficos. Revisión de la literatura. Int. J. Odontostomat. 2017; 11(1): 31-9. doi: http://dx.doi. org/10.4067/S0718-381X2017000100005

6. Hechavarria B, Venzant S, Carbonell M, Carbonell C. Salud bucal en la adolescencia. MEDISAN. 2013; 17(1): 117-25. Disponible en: http://scielo.sld.cu/scielo.php?script=sci_ arttext\&pid=S1029-30192013000100015\&lng=es.

7. Slade G. Derivation and validation of a short form oral health impact profile. Community Dent Oral Epidemiol. 1997; 25: 28490. doi: https://doi.org/10.1111/j.1600-0528.1997.tb00941.x

8. Montero-Martín J. Validation of the oral Health Impact Profile (OHIP-14 sp) for adults in Spain. Med Oral Patol Oral Cir Bucal 2009; 14(1): 44-50. PMID: 19114956.

9. De la Fuente J, Sumano O, Sifuentes M, Zelocuatecatl A. Impacto de la salud bucal en la calidad de vida de adultos mayores demandantes de atención dental. Univ Odontol. 2010; 29(63): 83-92. Disponible en: http://www.redalyc.org/articulo. oa?id=231216366010

10. Cohen F.; Souza R, Rebelo, M. A. B. Quality of life related to oral health: contribution from social factors. Ciênc. Saúde Coletiva, 2011; 16(1): 1007-15. doi: https://doi.org/10.1590/ s1413-81232011000700033

11. Espinoza M. Calidad de vida en adultos mayores portadores de prótesis dental del Distrito de Cascas. La Libertad - Perú. Oactiva. 2020; 6(1): 19-24. doi: https://doi.org/10.31984/ oactiva.v6i1.543x

12. Jenei Á, Sándor J, Hegedûs C, Bágyi K, Nagy L, Kiss C, Szabó G, Márton IJ. Oral health-related quality of life after prosthetic rehabilitation: a longitudinal study with the OHIP questionnaire. Health Qual Life Outcomes. 2015; 13: 99. doi: https://doi. org/10.1186/s12955-015-0289-2
13. Utsman R, Padilla M, Rodríguez L. Uso de la versión en español del perfil de impacto en la Salud Oral-14 para evaluar el impacto del tratamiento dental en la calidad de vida relacionada a la salud oral entre costarricenses. Revista Científica Odontológica. 2016; 12(2): 24-9. Disponible en: http://www.redalyc.org/articulo. oa?id=324250005004

14. Mohamed N, Saddki N, Yusoff A, Mat Jelani A. Association among oral symptoms, oral health-related quality of life, and health-related quality of life in a sample of adults living with HIV/AIDS in Malaysia. BMC Oral Health. 2017; 17: 119. doi: https://doi.org/10.1186/s12903-017-0409-y

15. Silva M, Villaça E, Magalhães C, Ferreira E. Impacto da perda dentária na qualidade de vida. Ciênc Saúde Coletiva 2010; 15(3): 841-50. doi: https://doi.org/10.1590/S141381232010000300027

16. Henriques J, Soares G, Hordonho P, Moura C, Santos E, De Souza Coelho R. Impacto del Edentulismo en la calidad de vida de individuos brasileños. Acta Odont. Venez. 2013; 51(3). Disponible en: https://www.actaodontologica.com/ ediciones/2013/3/art-9

17. Gaber, A, Galarneau, C, Feine, JS, Emami, E. Rural-urban disparity in oral health-related quality of life. Community Dent Oral Epidemiol. 2018; 46(2): 132-42. doi: https://doi. org/10.1111/cdoe.12344

18. Hanisch M, Wiemann S, Bohner L, Jung S, Kleinheinz J, Igelbrink S. Oral Health-Related Quality of Life in People with Achalasia. M (Kaunas). 2020; 56(6): 286 doi: https://doi. org/10.3390/medicina56060286

19. Papaioannou W, Oulis CJ, Latsou D, Yfantopoulos J. Oral health-related quality of life of greek adults: a cross-sectional study. Int J Dent. 2011; 2011: 360292. doi: https://doi. org/10.1155/2011/360292

20. Rodakowska E, Wilczyńska-Borawska M, Fryc J, Baginska J, Naumnik B. Oral health-related quality of life in patients undergoing chronic hemodialysis. Patient Prefer Adherence. 2018; 1(12): 955-61. doi: https://dx.doi.org/10.2147\%2FPPA. $\mathrm{S} 161638$

21. Koistinen S, Olai L, Stanhlnacke K, Falt A, Ehrenberg A. Oral healt-related quality of life and associated factors among older people in short-them care. Int J Dent Hyg. 2020; 18(2): 163-72. doi: https://doi.org/10.1111/idh.12424

22. Acharya S. Oral health-related quality of life and its associated factors in an Indian adut population. Oral Health Prev Dent. 2008; 6(3): 175-84. PMID: 19119571 